\title{
Rechtsgeschichte
}

http://www.rg-rechtsgeschichte.de/rg2

Zitiervorschlag: Rechtsgeschichte Rg 2 (2003)

$\operatorname{Rg} 22003 \quad 151-160$

http://dx.doi.org/10.12946/rg02/151-160

\section{Klaus Günther}

John Rawls (1921-2002)

Ein Restatement 


\section{John Rawls (I92I-2002) Ein Restatement}

Es war eine heißer, schwüler Sommerabend, als John Rawls im Hörsaal H der Frankfurter Universität einen Vortrag hielt. Er sprach leise, fast schleppend, und er hatte sich vorgenommen, den Text in einer deutschen Übersetzung vorzulesen, was für einen amerikanischen Professor ungewöhnlich war und deshalb Bewunderung verdiente. Doch war die angespannte Konzentration spürbar, die Rawls aufbringen musste, um deutsche Worte mit so wenig amerikanischer Phonetik wie möglich zu sprechen, und der Vortrag wurde dadurch noch langsamer, die Stimme noch leiser. Außerdem funktionierte das Mikrofon nicht richtig. Deshalb wurde es ihm von seinem Übersetzer, Wilfried Hinsch, mit ausgestrecktem Arm so nahe an den Mund gehalten, dass wenigstens ein paar Worte zu verstehen waren. Nach kurzer Zeit verließen die ersten Zuhörer den Hörsaal. Der ausgestreckte Arm des Helfers wurde sichtbar schwerer; Anstrengung und Hitze ließen Schweißbäche rinnen und das Oberhemd nass werden. Der Vortrag war nicht einfach. Rawls machte, wie gewohnt, keinerlei Konzessionen, sondern diktierte einen komplexen Satz nach dem anderen. Wer etwas verstehen wollte, musste von der komischen Situation absehen, alle Kräfte gegen die von der schwülen Hitze geführten Ermüdungsattacken aufbieten und sich irgendwie konzentrieren. Der einzige, der sich davon nicht beirren ließ, sondern hartnäckig Satz für Satz in den schwülen Sommerabend hämmerte, war der kleine, schmächtige, blasse, sein Gesicht hinter einer riesigen Brille verbergende, aber Respekt heischende Professor Rawls. Wenn Geist so unmittelbar präsent ist, wird eben alles andere banal.

Hartnäckigkeit ist vielleicht die treffendste Charakterisierung für die Art und Weise, in der er das eine große Theorieprojekt sein intellektuelles Leben lang verfolgte. In Abwandlung einer Anekdote über den ihm in dieser Hinsicht nicht unähnlichen Niklas Luhmann ließe es sich so zusammenfassen: Projekt »Theorie der Gerechtigkeit «, Laufzeit vierzig Jahre, Kosten keine. Der erste Aufsatz, der das Thema »Gerechtigkeit als Fairneß « anschlägt, stammt aus dem Jahre I958. ${ }^{\mathrm{I}}$ Alles weitere sind Ausarbeitungen, Ergänzungen, Präzisierungen, Korrekturen - aber kein Themenwechsel, keine Fundamentalkorrektur, kein Verwerfen früherer

\footnotetext{
I Dt. Übersetzung herausgegeben von Otfried Höffe: JoHn Rawls, Gerechtigkeit als Fairneß, Freiburg, München I977, 34 ff. Der Originaltext findet sich jetzt in der von Samuel Freeman edierten Ausgabe: JoHn Rawls, Collected Papers, Cambridge, Mass. I999, S. $47 \mathrm{ff}$.
} 
Thesen. Herausgeber von Sammelbänden verzweifelten regelmäßig, wenn sie einen Vortrag von Rawls veröffentlichen wollten, weil er ohne Rücksicht so lange an seinen Argumenten arbeitete, bis sie ihm gut genug begründet erschienen. »Justice as Fairness - A Restatement " heißt nicht zufällig die letzte seiner zu Lebzeiten erschienenen Veröffentlichungen. ${ }^{2}$ Mehr als vierzig Jahre lang: $R e$ statement. Wer sein Leben auf eine Sache verwendet, muss von der Richtigkeit seiner Intuition überzeugt und gleichzeitig von einem fast unheimlichen Perfektionsdrang beseelt sein. Dass es aber keine bornierte Idiosynkrasie war, die ihn antrieb, einen komplexen Gedanken über Jahrzehnte auszubreiten, zeigt sein großer internationaler Erfolg.

Das diesen Erfolg begründende Werk war die I97I erschienene » Theory of Justice «. ${ }^{3}$ Im Rückblick vermag man kaum noch zu verstehen, warum es die philosophische Welt in zuerst zögerndes und skeptisches, dann aber um so nachhaltigeres Staunen versetzte. Alle damaligen Rezensionen und alle jüngsten Nachrufe heben einstimmig hervor, dass mit diesem Buch zum ersten Mal nach langer Zeit wieder eine materiale Gerechtigkeitstheorie vorgelegt worden sei, welche sich um die Begründung praktischer Prinzipien bemühe, anstatt sich auf metaethische und formale Fragen zu beschränken. Diese Einschätzung trifft freilich nur zum Teil zu. Gewiss - es schien zu dieser Zeit wichtiger, die Sprache der Moral zu untersuchen als über Moral selbst zu reden, die möglichen Bedeutungen des Prädikates "gut « zu analysieren statt zu überlegen, welche Handlungen und Zustände denn nun eigentlich "gut " genannt zu werden verdienten. Aber in dieser von der analytischen Philosophie gepflegten und kultivierten Methode zeigte sich selbst eine ethische Tugend, die in unklarem Sprechen eine Ursache für moralisches Versagen vermutete - und dafür in den ideologischen Verwirrungen der Zeit auch genügend Beweise fand. Rawls hat die von der analytischen Philosophie gesetzten Standards keineswegs unterboten oder verworfen, sondern sich selbst zu eigen gemacht. Das mochte einer der Gründe dafür sein, dass die " Theory of Justice" nicht weniger als 600 Seiten umfasst.

Notwendig war dieser Umfang jedoch vor allem aus einem weiteren Grund: Die damalige praktische Philosophie befand sich nicht nur wegen des Übergewichts der Metaethik in einer Krise, sondern auch, weil der Geltungsanspruch moralischer Überzeugungen überhaupt fragwürdig schien. Hatten praktische Fragen in

\footnotetext{
2 Hg. von Erin Kelly, Cambridge,

Mass. 200I.

3 Cambridge, Mass. I97I. I999

erschien eine "Revised Edition",

Cambridge, Mass. u. Oxford.
} 
der wissenschaftlich-technischen Welt überhaupt noch einen Sinn, der über technisch lösbare Probleme hinaus ging? Waren moralische Überzeugungen mehr als nur der Ausdruck subjektiver Wertschätzungen, die letztlich in irrationalen individuellen Entscheidungen wurzelten, aber keineswegs so vernünftig begründet werden konnten wie die Sätze der Naturwissenschaften? Wenn diesen Wertschätzungen überhaupt ein Sinn abzugewinnen war, bestand er dann nicht vor allem in dem Wunsch nach individuellem und kollektivem Wohlergehen, so dass sich vermuten ließ, der Utilitarismus sei faktisch die am weitesten verbreitete Moral? Sollte sich die Politik daher nicht auf die Ermöglichung wirtschaftlichen Wohlstands für möglichst viele durch einen freien Markt mit sozialem Ausgleich beschränken? Wer in dieser Situation gegen die Krise der praktischen Philosophie anschreiben wollte, musste sich mit Argumenten wappnen. Rawls hat dies getan. Das Buch enthält von der ersten bis zur letzten Seite keinen einzigen überflüssigen, bloß ornamentalen, den Leser einfangen wollenden Satz. Ein durchdachtes Argument folgt auf das andere, jede Behauptung wird begründet, Konsequenzen werden geduldig ausbuchstabiert, mögliche Einwände erwogen und kritisch diskutiert, Alternativen gesucht und mit Gründen verworfen. Man konnte also wieder praktisch philosophieren, aber Rawls hatte die standards, die man dabei einhalten musste, fast unerreichbar hoch gelegt.

Wer sich dann doch an die langwierige und höchste Konzentration abfordernde Lektüre machte, wurde überrascht von der Einfachheit und Klarheit der Gerechtigkeitsprinzipien, die Rawls zu begründen unternommen hatte. Dass in einer gerechten Gesellschaft das größtmögliche Maß an Freiheitsrechten zu gewähren sei, das mit dem gleichen Freiheitsrecht eines jeden Einzelnen vereinbar ist - dieses Prinzip war in mehr oder weniger ähnlich lautenden Varianten bereits bekannt. Anders verhielt es sich mit dem zweiten Prinzip, nach dem soziale und wirtschaftliche Ungleichheiten gegenüber einer allgemeinen Gleichheit nur dann gerechtfertigt seien, wenn es den am meisten Benachteiligten in einem System der Ungleichheit besser ergehe als in einem System der Gleichheit. Um Verteilungsgerechtigkeit hatten sich die praktische und die politische Philosophie lange nicht gekümmert, seitdem die Güterverteilung im Wesentlichen dem freien Markt überlassen worden war und der Sozialstaat weniger als eine Forderung der Gerechtigkeit, sondern als Lösung von Funktionsproblemem des Marktes 
verstanden wurde. Rawls hatte mit diesen beiden Prinzipien und dem Postulat des Vorrangs des ersten vor dem zweiten Prinzip den sozialstaatlichen Kompromiss, von dem die westliche Welt nach dem Zweiten Weltkrieg in unterschiedlichen Formen geprägt war, philosophisch am klarsten und konsequentesten auf den Begriff gebracht. Zugleich provozierte die strikte Verpflichtung auf das gleiche Recht jedes Einzelnen und auf das individuelle Wohlergehen den in der anglo-amerikanischen Welt vorherrschenden Utilitarismus.

Es waren jedoch weniger diese beiden Prinzipien, mit denen Rawls Aufsehen erregte, als vielmehr die Art und Weise ihrer Begründung. Die Krise der praktischen Philosophie beruhte nicht zuletzt auf der allgemeinen Überzeugung, dass Gerechtigkeitsprinzipien entweder nur metaphysisch zu begründen seien - was den Rationalitätsansprüchen der modernen Wissenschaft widersprach - oder in letzter Instanz gar nicht begründet werden könnten, sondern aus einer individuellen, nicht weiter ergründbaren Entscheidung hervorgingen. Vordergründig schien es so, als habe Rawls als Vehikel für eine rationale Begründung nur das altbekannte Modell des Vertrages wiederbelebt. Dass die beiden Gerechtigkeitsprinzipien das Ergebnis eines Vertragsschlusses in einem Urzustand seien, in dem die Parteien durch den berühmten »veil of ignorance " (Schleier des Nichtwissens) daran gehindert sind, vorherzusehen, welche Position sie in der zu ordnenden Gesellschaft einnehmen würden, also das Ergebnis eines Unparteilichkeit garantierenden Entscheidungsverfahrens - mit diesem Begründungsweg schien Rawls alte Motive wieder aufzunehmen, sie aber mit den modernsten methodischen Mitteln, unter anderem der ökonomischen Entscheidungstheorie, gegen den Verdacht abzuschirmen, hier werde zugleich eine alte Vernunftmetaphysik wiederbelebt. Zudem konnte Rawls an die alte Einsicht der Vertragstheoretiker anknüpfen, dass die grundlegenden Normen einer Gesellschaft mit den individuellen Interessen eines jeden Einzelnen vereinbar sein müssen.

Noch viel aufregender und auch folgenreicher war jedoch der zweite Begründungsweg, den Rawls beschritt. Der Vertragsschluss im Urzustand sollte nur ein Vehikel sein, »a device of representation «, wie er es später nannte, ein Hilfsmittel für das Verstehen. Das eigentliche Hauptgewicht legte Rawls auf die Begründung der Voraussetzungen des Urzustands, insbesondere der Anforderun- 
gen, die an die Parteien, ihr Wissen und ihre Fähigkeiten zu stellen seien. Wenn diese Voraussetzungen nicht ihrerseits wiederum in einem Urzustand gerechtfertigt werden können, dann bliebe, so durfte man befürchten, nur der Weg in die Metaphysik übrig. Rawls' Innovation bestand darin, ein alternatives Begründungsmodell vorzuschlagen, das im Wesentlichen darin besteht, die Gerechtigkeitsprinzipien auf eine Überlegungsprozedur zu gründen, durch welche Personen, die ein Interesse daran haben, miteinander in einem System der fairen Kooperation zu leben, zu wohlüberlegten Urteilen gelangen. Wer nur vernünftig überlegt, wer sich auf seine moralischen Intuitionen besinnt und auf die praktischen Urteile, die er oder sie schon für richtig hält, und diese miteinander vergleicht und korrigiert, könnte auch auf jene beiden Prinzipien kommen. Diese Methode des »reflective equilibrium « (Überlegungsgleichgewicht) klingt einerseits ungeheuer trivial und nichtssagend, aber sie ist, wie die detaillierten Ausführungen in dem Buch belegen, in Wahrheit äußerst komplex und anspruchsvoll. Gewonnen wurde damit nichts weniger als ein immanenter, an den vorhandenen Interessen und Fähigkeiten der Einzelnen ausgerichteter Begründungsweg, der ohne metaphysische Prämissen auskommt.

Diesem Programm blieb Rawls im Folgenden treu, und man könnte seine weiteren Arbeiten und Reaktionen auf kritische Einwände als das kontinuierliche Ausbuchstabieren dieses Programms lesen. Dies gilt insbesondere für die späteren Veröffentlichungen, in denen Rawls wie kaum ein anderer politischer Philosoph das »Faktum des Pluralismus « ernst nahm, um die Prinzipien der Gerechtigkeit für eine pluralistische Welt so zu rechtfertigen, dass der Pluralismus dabei nicht verschwindet. »Justice as Fairness: Political not Metaphysical « - dieser Aufsatztitel fasst bündig zusammen, wie Rawls den von ihm eingeschlagenen Begründungsweg verstanden wissen wollte: ${ }^{4}$ als eine politische Theorie der Gerechtigkeit, die von niemandem verlangt, die eigene Identität aufzugeben. Noch bevor die Diskussion über Globalisierung richtig in Gang kam, konfrontierte sich Rawls bereits mit dem Problem, wie Gerechtigkeit als Fairness in einer Welt zu verwirklichen sei, in der es Gesellschaften und Staaten mit unterschiedlichen Identitäten und Weltanschauungen gibt. ${ }^{5}$

Anders als in Harvard und Oxford hat es hierzulande sehr lange gedauert, bis nach dem Erscheinen der deutschen Übersetzung der »Theorie der Gerechtigkeit« im Jahre 1975 die Aus-

4 In: John Rawls, Collected Papers

(Fn. I) 388. Dt. Übers. in: JoHN

Rawls, Die Idee des Politischen

Liberalismus - Aufsätze I978-

I989, hg. v. WiLfRIED Hinsch, Frankfurt a. M. I992, $255 \mathrm{ff}$.

5 John Rawls, The Law of Peoples, Cambridge, Mass. I999.

Dt. Übers.: Das Recht der Völker, Berlin, New York 2002. 
einandersetzung mit Rawls begann. Auch jetzt noch bleibt die Rezeption eher zurückhaltend, zumeist beschränkt auf respektvolle Darstellungen und Zusammenfassungen. Der Verdacht liegt nahe, dass viele den 600 schwierigen Seiten eben doch nicht gewachsen sind, sondern sich auf selektive Lektüre, sekundäre Darstellungen und Schlagworte verlassen. Dabei befand sich die praktische Philosophie Ende der sechziger Jahre auch hier in einer Krise, die noch verstärkt wurde durch den Anspruch, Moral so weit wie möglich durch Gesellschaftstheorie wenn nicht zu ersetzen, so doch zumindest zu »entlarven «. Damit Gesellschaft funktioniert, sollte es weniger auf normative Orientierung ankommen als auf die Erkenntnis der überindividuellen Funktionszusammenhänge, die dann durch rationale Planung politisch zu steuern waren. Zwar hatten die besseren Gesellschaftstheoretiker schon immer gewusst, dass das eine ohne das andere nicht zu haben ist, aber solche komplexeren Theorien steckten allenfalls in den Anfängen. Daneben hatte die deutsche geistesgeschichtliche Tradition moralische Fragen weitgehend zugunsten eines hermeneutischen Einrückens in das Überlieferungsgeschehen neutralisiert, das vor dem »abstrakten Sollen « ängstlich zurückschreckte. Schließlich war die analytische Wissenschaftstheorie auch hier in der Gestalt des Kritischen Rationalismus breit rezipiert worden, mit den bekannten Behauptungen, dass nur falsifizierbare deskriptive Aussagen über die objektive Welt rational kritisierbar seien, dass praktische Sätze entweder nur als deskriptive Aussagen über faktisch vorhandene Wertüberzeugungen oder als falsifizierbare Zweck-Mittel-Hypothesen darunter fielen, ansonsten aber Wertüberzeugungen nur das Ergebnis letztlich irrationaler individueller Entscheidungen seien. In dem selben Jahr, als Rawls' »Theory of Justice « erschien, gab Manfred Riedel ein mehrbändiges Sammelwerk mit dem programmatisch gemeinten Titel »Rehabilitierung der praktischen Philosophie« heraus. Der Unterscheid konnte größer freilich nicht sein. Während Rawls nur lose an die moralphilosophische Tradition anknüpfte, um dann einen originellen, nach-metaphysischen Begründungsweg zu eröffnen, verblieben die deutschen Autoren viel tiefer in dieser Tradition, führten die alten Debatten zwischen Kant und Hegel über das abstrakte Sollen weiter und bemühten sich, wie schon der Titel jenes Sammelwerks ankündigte, vor allem um eine Aktualisierung aristotelischer Motive. Otfried Höffe kam das Verdienst zu, als einer der ersten die »Theorie der Gerechtigkeit« in die 
deutsche Diskussion einzuführen. Freilich blieb der von ihm herausgegebene Sammelband (mit - von der Einleitung Höffes abgesehen - ausschließlich englischsprachigen Autoren) ebenfalls zunächst ohne größere Resonanz. ${ }^{6}$

Dabei hätte eine deutsche Rezeption sehr schnell ein anschlussfähiges Motiv finden können, das später von Rawls selbst immer deutlicher akzentuiert und elaboriert wurde. Rawls war dem Utilitarismus bei seinem Versuch, ihn zu überwinden, so weit entgegengekommen, dass er das Interesse jedes Einzelnen an seinem individuellen Wohlergehen als Kriterium für die Akzeptabilität der Gerechtigkeitsprinzipien bewahren wollte. Insoweit unterstellte er den am Urzustand beteiligten Personen Rationalität im Sinne der Zweckrationalität. Sollte das Ergebnis des Entscheidungsverfahrens jedoch zugleich unparteilich sein, musste jenes intersubjektive Kriterium hinzugenommen werden, das in der Kantischen Tradition der Moralphilosophie als (praktische) Vernunft reformuliert wurde. Der Vertragsschluss im Urzustand hinter einem Schleier des Nichtwissens sollte beide Kriterien vereinigen. Gleichwohl blieb das Verhältnis zwischen » rational « und » reasonable « noch unklar. In seinen Abhandlungen über »Kantian Constructivism in Moral Theory « versuchte Rawls, das Kantische Motiv stärker zur Geltung zu bringen. ${ }^{7}$ Die posthum veröffentlichten Vorlesungen zur Geschichte der Moralphilosophie belegen, mit welcher Intensität Rawls sich über lange Zeit mit Kant und dessen Vorgängern Hume und Leibniz sowie mit dem kritischen Nachfolger Hegel auseinander gesetzt hat. ${ }^{8}$

Richtig in Gang kam die deutsche Rezeption erst angesichts einer Kontroverse, die durch eine schmale Dissertation eines bis dahin unbekannten jungen Autors ausgelöst wurde. I982 veröffentlichte Michael Sandel seine Abhandlung »Liberalism and the Limits of Justice ", mit der eine intellektuelle Bewegung begründet wurde, die unter dem Titel des »Kommunitarismus « schnell berühmt geworden ist. ${ }^{9}$ Ohne hermeneutischen Ballast, wie dies zur Zeit wohl nur in England und in den USA möglich ist, aktualisierte Sandel die Einwände, die Aristoteles bereits gegen Plato, Hegel gegen Kant und Durkheim gegen die Vertragstheorien geführt hatten. Die Gründung der gesellschaftlichen Grundordnung auf ein unparteiliches Entscheidungsverfahren - wie der Vertragsschluss im Urzustand hinter dem Schleier des Nichtwissens operiere mit fiktiven Personen, die von ihren konkreten Lebens-

6 Über John Rawls' Theorie der Gerechtigkeit, hg. v. OTFRIED HöFFE, Frankfurt a. M. I977. In der spezifisch rechtsphilosophischen Rezeption hat es damals nur eine ausführliche und scharfsinnige kritische Rezension gegeben: Gertrude LübBe, Die Auferstehung des Sozialvertrags - John Rawls' Gerechtigkeitstheorie, in: Rechtstheorie 8 (I977) I 85 ff.
7 In: John Rawls, Collected Papers (Fn. I) 303 ff. Dt. Übers. in: JoHN Rawls, Die Idee des politischen Liberalismus (Fn. I) 80 ff. Rawls hatte seine Argumente in dem Buch Political Liberalism, New York I993, 89 ff. (Dt. Übers.: Politischer Liberalismus, Frankfurt a. M. I998) noch einmal präzisiert.
8 John Rawls, Geschichte der Moralphilosophie, Frankfurt a. M. 2002.

9 Cambridge, UK 1982. 
bezügen, ihrer individuellen Identität, ihren immer schon vorgegebenen kollektiven Zugehörigkeiten abstrahieren müssten. Die von Sandel angeschlagenen Themen bestimmten die folgende Debatte und nötigten Rawls zu immer wieder neuen Restatements. Freilich wäre dies kaum möglich gewesen, wenn Rawls' Theorie der Gerechtigkeit nicht selbst schon so reichhaltig gewesen wäre, dass sie überall Anknüpfungspunkte bot. So gibt es denn auch kaum einen Autor oder eine Autorin in dieser Debatte, der oder die sich nicht auf Rawls bezieht - wie Alasdair MacIntyre, Michael Walzer, Charles Taylor, Joshua Cohen. Die Vermutung dürfte nicht übertrieben sein, dass erst mit dieser Kontroverse Rawls einem größeren deutschen Publikum bekannt wurde. Deren Motive waren den meisten eben schon aus dem Projekt der »Rehabilitierung der praktischen Philosophie« vertraut. Jürgen Habermas hatte sich zwar schon vorher an der Auseinandersetzung mit Rawls über das Verhältnis von » rational « und » reasonable « beteiligt, fand sich aber in der Kommunitarismusdebatte plötzlich in einem Boot mit Rawls, weil die Diskurstheorie der Moral immer schon mit ähnlichen Einwänden konfrontiert worden war. Der wohl beste deutsche Beitrag zu dieser Debatte ist denn auch aus einer produktiven Rezeption von Rawls und Habermas hervorgegangen: Rainer Forsts Buch »Kontexte der Gerechtigkeit «. ${ }^{\text {Io }}$

Rawls' Versuch, die Einwände des Kommunitarismus aufzunehmen, ohne seine Intuition von der Gerechtigkeit als Fairness und von dem Vorrang des Rechten vor dem Guten ${ }^{\text {II }}$ preiszugeben, führte freilich in eine neue Kontroverse. Rawls verstand seine Theorie radikal antimetaphysisch und rein politisch. Aber mit der Idee einer Rechtfertigung durch öffentlichen Vernunftgebrauch hielt er weiterhin an der kantischen Tradition fest und bot damit zugleich auch Anknüpfungspunkte für die Diskurstheorie des Rechts von Jürgen Habermas. Über die Frage allerdings, ob sich das Kriterium des öffentlichen Vernunftgebrauchs als eine rein politische Theorie begründen ließe, ohne darüber nichts mehr als nur eine partikulare, interne Selbstverständigung der nordamerikanischen Gesellschaft über ihre politischen Grundüberzeugungen zu werden, musste es zu einer umfangreichen Kontroverse kommen. Rawls warf Habermas vor, mit der Diskurstheorie doch eine "umfassende Weltsicht " (comprehensive doctrine) zu behaupten, die sich letztlich auf in einer pluralistischen Welt nicht verallgemeinerbare metaphysische Prämissen stütze, während Habermas

IO RAINER Forst, Kontexte der Gerechtigkeit - Politische Philosophie jenseits von Kommunitarismus und Liberalismus, Frankfurt a. M. I994.

I I JOHn Rawls, The Priority of Right and Ideas of the Good, in: DERs., Collected Papers (Fn. I) 449 ff., dt. Übers. in: DERs., Die Idee des politischen Liberalismus (Fn. I) 364 ff. Präzisierungen wie- derum in: DERs., Political Liberalism (Fn. 7) I73 ff. 
die Gefahr beschwor, dass eine politische Theorie der Gerechtigkeit, die auf philosophische Begründung verzichtet, nur noch als partikularer Ausdruck einer historisch kontingenten politischen Identität ohne Geltungsanspruch für Nichtmitglieder verstanden werden könnte. ${ }^{\text {I2 }}$

Dies ist nur eines der kontroversen Themen, die nach Rawls' Tod offen geblieben sind. Ein anderes Thema beginnt erst langsam wieder Aktualität zu gewinnen: die distributive Gerechtigkeit. Angesichts der aktuellen Krise des Sozialstaates und einigen Versuchen, »soziale Gerechtigkeit « als Unwort aus dem politischen Vokabular zu verbannen, lässt sich bei Rawls ein reichhaltiges Arsenal an Gegenargumenten finden. Die Frage etwa, ob und inwieweit natürliche Begabungen oder Fähigkeiten, die man nur unter glücklichen, aber ungleich verteilten sozialen Umständen auszubilden vermag, in die Entscheidung über die Grundordnung einer Gesellschaft einfließen dürfen, wird von Rawls unter dem Stichwort der »natürlichen Lotterie « ausführlich diskutiert. Dies hatte schon vor längerer Zeit Robert Nozick zu einem liberalistischen Gegenentwurf veranlasst. ${ }^{\mathbf{} 3}$ Inzwischen ist diese Debatte über Kriterien gerechter Güterverteilung wieder aufgenommen worden, unter anderem durch Ronald Dworkin und Amartya Sen. ${ }^{\mathbf{I}}$ Weitere wichtige Debatten - etwa über die Frage, wie sich der Kantianische Konstruktivismus zur postmodernen Kritik am Subjekt verhält - stehen noch aus. Fündig wird allemal, wer nur die Mühe auf sich nimmt, 600 anspruchsvolle Seiten (und weitere Veröffentlichungen) zu lesen.

Dies scheint sogar dem 2. Senat des Bundesverfassungsgerichts gelungen zu sein, als es im Beschluß zum Länderfinanzausgleich aus dem Jahre I999 dem Gesetzgeber im 3. Leitsatz auftrug: »Die Finanzverfassung verlangt eine gesetzliche Maßstabgebung, die den rechtsstaatlichen Auftrag eines gesetzlichen Vorgriffs in die Zukunft in der Weise erfüllt, dass die Maßstäbe der Steuerzuteilung und des Finanzausgleichs bereits gebildet sind, bevor deren spätere Wirkungen konkret bekannt werden. « ${ }^{\mathrm{I} 5}$ Besser könnte man nicht ausdrücken, was mit der Idee eines unparteilichen Entscheidungsverfahrens hinter dem Schleier des Nichtwissens gemeint ist.

Dass Rawls in dieser Entscheidung keine Erwähnung findet, hätte ihn vermutlich am wenigsten gestört, da er ja nur reformulieren wollte, was jeder Einzelne im Zustand des Überlegungsgleichgewichts ohnehin wissen könnte. Dass man sich den Weg in

I 2 JÜRgEN HABERMAS, Reconciliation through the Public Use of Reason: Remarks on John Rawls' Political Liberalism, in: Journal of Philosophy 42 (I995) I09 ff.; John Rawls, Reply to Habermas, in: Journal of Philosophy 42 (I995) S. I32 ff.

I 3 Robert Nozick, Anarchy, State, Utopia, Oxford I974.

I4 Vgl. G. A. Cohen, On the Currency of Egalitarian Justice, in:
Ethics 99 (I989), 906 ff.; JoHn E. Roemet, A Pragmatic Theory of Responsibility for the Egalitarian Planner, in: Philosophy \& Public Affairs 22 (I993) I $46 \mathrm{ff}$. I 5 BVerfGE IOI, I $58 \mathrm{ff}$. 
diesen Zustand indes nicht als eine humorlose und langweilige Veranstaltung in Seminarräumen vorstellen muss, verrät ein kleiner Hinweis, den Rawls in der Einleitung zu einem seiner Bücher gibt. Er rückt den Autor zudem auch wieder ein wenig in die Nähe der conditio humana. Dort bedankt er sich bei seinen Freunden Ronald Dworkin und Thomas Nagel für eine »rare illuminating midnight conversation in the deserted bar of the Santa Lucia Hotel in Napoli in June I988. ${ }^{16}$ Zuweilen braucht der Geist solche Hilfsmittel, um präsent zu werden.

Klaus Günther

I 6 John Rawls, Political Liberalism

(Fn. 7), S. xxxi. 